\title{
Bayesian Hierarchical Model of Width of Keratinized Gingiva
}

\author{
Veska Noncheva ${ }^{1}$, Maria Dobreva $^{1}$, Ivan Chenchev $^{2}$ \\ ${ }^{\text {I}(F a c u l t y ~ o f ~ M a t h e m a t i c s ~ a n d ~ I n f o r m a t i c s, ~ U n i v e r s i t y ~ o f ~ P l o v d i v ~ " P a i s i i ~ H i l e n d a r s k i ", ~} 24$ Tzar Asen, 4000, \\ Plovdiv, Bulgaria) \\ ${ }_{2}^{2}$ (Department of Oral Surgery, Faculty of Dental Medicine, Medical University-Plovdiv, Bulgaria)
}

\begin{abstract}
The purpose of this paper is to offer a method for studying the treatment result of gingival recession. A parameter showing the success of the surgical treatment of gingival recessions is the keratinized gingival width. It was measured four times: at baseline, after 1 month, after 3 months and after 6 months. Every patient has data that can be described by an individual trend. Bayesian hierarchical model of the keratinized gingiva width's increase rate is built.
\end{abstract}

Keywords: Biostatistics, gingival recession, hierarchical modeling, longitudinal data, split-mouth randomized clinical trial

\section{Introduction}

The amount of keratinized gingiva is derived from the sum of the free gingiva and attached gingiva. Keratinized gingiva width $(\mathrm{KGW})$ is measured from the mucogingival junction (MGJ) to the lowest point of the gingival margin in the middle of the crown of the tooth with gingival recession. In a healthy and intact periodontium, the length of keratinized attached gingiva includes coronally from the bottom of gingival sulcus to the mucogingival junction apically. The width of attached gingiva varies with the age. The keratinized attached gingiva provides the periodontium with increase resistance to external injury and stabilized the gingival margin against physical forces and helps patients plaque control measurements. An adequate amount of keratinized gingiva maintains gingival health by protecting the marginal gingiva from bacterial invasion [3,11], preventing an increase in gingival recession [4,22], facilitating plaque control [3,5,6,23], and improving denture stability $[12,23]$

Ainamo et al. [11] in different studies said that, MGJ remains stationary throughout life and changes in width of attached gingiva are caused by modification in position of coronal gingival margin. The width of attached gingiva increases with age and in supra-erupted teeth [7,15]. Lang and Löe [5] reported a study on the relationship between the gingival width and inflammation, in an effort to determine the adequate amount: In $100 \%$ of teeth with less than $2 \mathrm{~mm}$ of keratinized tissue, inflammation and exudates was present; $76 \%$ of cases with greater than $2 \mathrm{~mm}$ of keratinized tissue there was no exudates and was considered as clinically healthy. They concluded that $2 \mathrm{~mm}$ of keratinized gingiva, with less than $1 \mathrm{~mm}$ of attached gingiva is adequate to maintain gingival health. An adequate band of attached gingiva could be defined as that amount which is sufficient to prevent recession in opinion of individual practioners [16]. Thus no minimum width of attached gingiva has been established as standard necessary for gingival health. Another paper by Miyasato et al. [9] concluded that there is no relationship between inflammation and amount of attached gingiva whether or not plaque is present. According to Miller PD and Harris RJ one of the criteria to achieve complete root coverage is to achieve sufficient width of keratinized gingiva $(\mathrm{KGW}),[10,13]$. In the dental researches increasing $\mathrm{KGW}$ is an important criterion for determining the success of the treatment of gingival recession.

\section{Problem}

The aim of this paper is to present an approach for studying the rate of increase of the keratinized gingiva width after surgery. This research involved 30 people at the age of 23 to 70 years with a total of 118 symmetrical Miller's Class I and Class II gingival recessions on different places of the jaws. All of the patients' gingival recessions were treated surgically and the results were monitored for six months postoperatively. The recessions on one side of the jaw were treated with coronally advanced flap (CAF) combined with platelet rich fibrin membrane, while the other side was treated with CAF combined with connective tissue graft [1]. An approach for the comparison of two methods of treatment of gingival recessions is described in [24]. The success of the operation was evaluated through the measurement of the keratinized gingival width in millimeters. KGW was measured 4 times: at baseline, after 1 month, after 3 months and after 6 months. Every patient has data that can be described by an individual trend. Because of it we will build hierarchical model (see $[20,21]$ ) of the rate of increase of the keratinized gingiva width. Hierarchical models of phenomena from oral surgery are described in $[18,24]$. 


\section{Model}

We suppose that every individual $j$ contributes multiple observations of $\left(x_{i j}, y_{i j}\right)$ pairs, where the subscript notation $i \mid j$ means the $i$-th observation within the $j$-th individual (patient). The observations $y_{i j}, i=1$, $\ldots, 118, j=1,2,3$, are the values of KGW measured at $1^{\text {st }}, 3^{\text {rd }}$ and $6^{\text {th }}$ month after, and $x_{i j}$ are the values of KGW measured before, at baseline, $1^{\text {st }}$ and $3^{\text {rd }}$ month respectively. With these longitudinal data, we will estimate a regression curve for every individual. Our goal is to describe each individual with a linear regression, and simultaneously to estimate the typical slope and intercept of the group overall. A key assumption for our analysis is that each individual is representative of the group. Therefore, every individual informs the estimate of the group slope and intercept, which in turn inform the estimates of all the individual slopes and intercepts. Thereby we get sharing of information across individuals, and shrinkage of individual estimates toward the overarching mode.

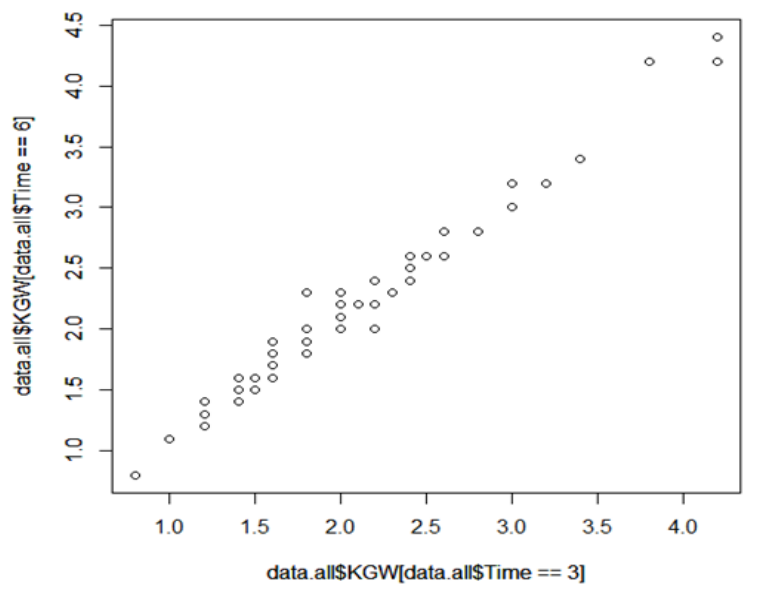

Figure 1.KGW data 3 months and 6 months after surgery.

The model assumes homogeneity of variance. Homogeneity of variance is less easy to identify visually when the predictor's values are not uniformly distributed. The apparent vertical spread of the data seems to be larger at $\mathrm{x}=1.5$ than at $\mathrm{x}=3.5$ in Figure 1. Despite this deceiving appearance, the data do respect homogeneity of variance. The reason for the apparent violation is that for regions in which $x$ is sparse, there is less opportunity for the sampled $y$ values to come from the tails of the noise distribution. In regions where the predictor is dense, there are many opportunities for the predicted variable to come from the tails. The data have outliers, and the use of heavy-tailed noise distributions is straight forward in contemporary Bayesian software. Student's t-distribution and its usefulness for describing data that might have outliers are perfectly explained in [21]. Student's t-distribution has the following three parameters: $\mu$ that controls its mean, the "scale" parameter $\sigma$ that controls its width, and a third parameter $v$ that controls the heaviness of its tails, called "normality" parameter. The parameter $v$ is also known as "degrees of freedom". The normality parameter can range continuously from 1 to $\infty$. When $v=1$ the $\mathrm{t}$ distribution has very heavy tails and it is a convenient descriptive model of data with outliers. When $v$ approaches $\infty$ the $t$ distribution becomes much closed to normal.

\subsection{Hierarchical dependency structure}

The hierarchical dependencies in the model are presented in Figure 2.

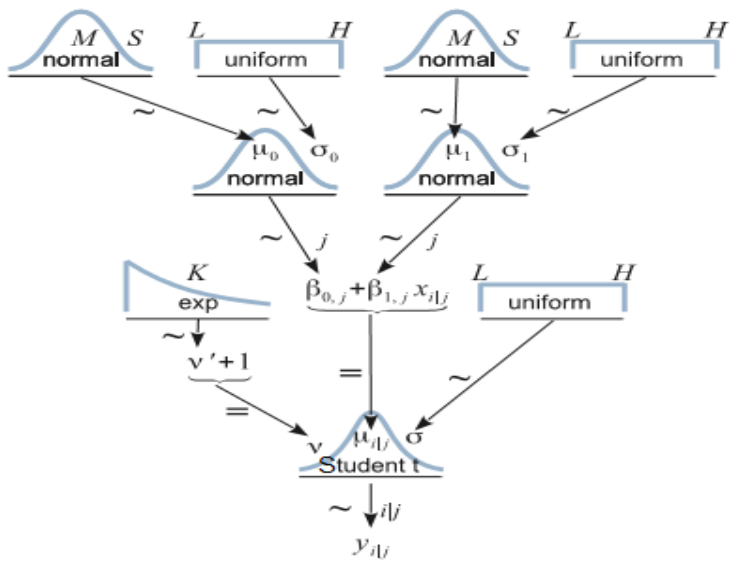

Figure 2. A model of dependencies. The standard deviation of the noise within each subject is the same for all subjects. 
The datum $y_{i j j}$ is the $i$-th datum within the $j$-th subject. It has a Student's $t$-distributed random value around the central tendency $\mu_{i \mid j}=\beta_{0, j}+\beta_{1, j} x_{i \mid j}, j=1,2,3, i=1, \ldots, 118$. The intercept and slope are subscripted with $j$, meaning that every subject has its own slope and intercept. The slopes across the individuals all come from a higher-level normal distribution. The model assumes that $\beta_{1, j} \sim$ normal $\left(\mu_{1}, \sigma_{1}\right)$, where $\mu_{1}$ describes the typical slope of the individuals and $\sigma_{1}$ describes the variability of those individual slopes. Analogous considerations apply to the intercepts $\beta_{0, j} \sim$ normal $\left(\mu_{0}, \sigma_{0}\right)$. The group-level parameters are given generic vague priors. This model assumes that the standard deviation of the noise within each subject is the same for all subjects. The tdistribution's scale parameter $\sigma$ is given a noncommittal uniform prior, and the normality parameter $v$ is given a broad exponential prior. The intercept and slope are given broad normal priors that are vague on the scale of the data. The two group-level parameters $\beta_{0}$ and $\beta_{1}$ have separate normal priors. Our goal is to determine what combinations of the parameters are credible, given the data. The solution comes from Bayes' rule and its implementation in JAGS software [25]. Our task is to specify sensible priors and to make sure that the MCMC process generates a trustworthy posterior sample that is converged and well mixed.

\section{Solution}

The upper-right panel of Figure 3 shows the posterior distribution of the jointly credible values of the slope $\beta_{1,3}$ and the intercept $\beta_{0,3}$. The correlation of the credible values of the slope and intercept is extremely strong. This narrow diagonal posterior distribution is difficult for Gibbs sampling algorithms to explore, resulting in extreme inefficiency in the chains. The two parameter values change very slowly, and the MCMC chain is highly auto correlated. In practice, this requires us to wait too long before getting a suitably representative sample from the posterior distribution. In order to make the sampling more efficient, the data are standardized before being sent to the model. In this way credible regression lines do not suffer such strong correlation between their slopes and intercepts.

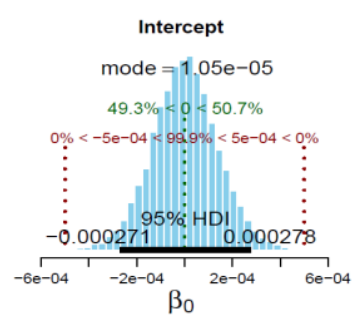

Scale

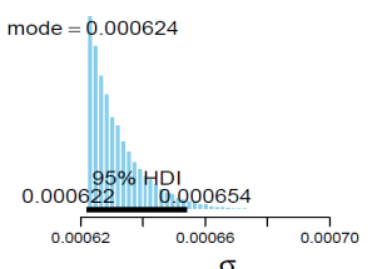

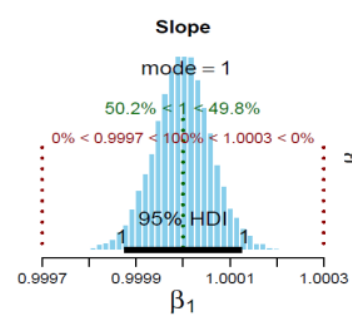
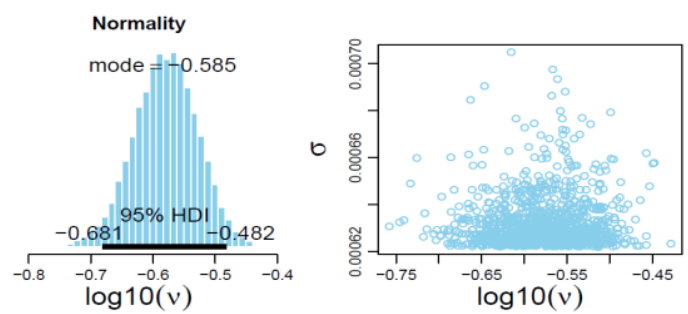

Figure 3.Marginal posterior distributions on parameters.
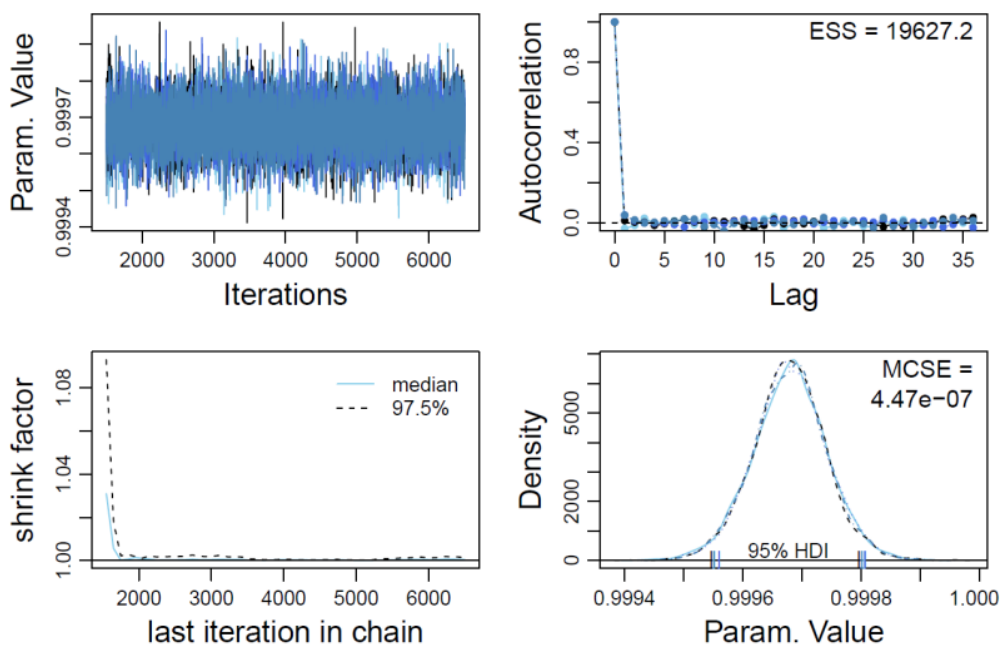

Figure 4. Convergence diagnostics for the standardized $\beta_{1,3}$ parameter (output of JAGS). 


\subsection{MCMC diagnostics}

Our first task is to check whether the chains appear to be well mixed and suitably representative of the posterior distribution. Figure 4 shows diagnostic information for the standardized $\beta_{1,3}$ parameter. The density plot in the lower-right shows that the three sub chains are well super-imposed which is echoed by the GelmanRubin statistic in the lower-left panel being very close to 1.0. The autocorrelation plot in the upper-right panel shows essentially zero autocorrelation. The diagnostic information for other parameters is similar.

Table 1. Estimates of the model parameters $v, \sigma, \beta_{0 \mathrm{k}}$ and $\beta_{1 \mathrm{k}}, \mathrm{k}=1,2,3$.

\begin{tabular}{|l|l|l|l|l|l|}
\hline & Mean & Median & Mode & $\begin{array}{l}\text { 95\% } \\
\text { HDIlow }\end{array}$ & $\begin{array}{l}\text { 95\% } \\
\text { HDIhigh }\end{array}$ \\
\hline beta0[1] & 1.40000 & 1.40000 & 1.60004 & 1.19982 & 1.60015 \\
\hline beta0[2] & 0.00000 & 0.00000 & 0.00000 & -0.00023 & 0.00022 \\
\hline beta0[3] & 0.00000 & 0.00000 & 0.00001 & -0.00024 & 0.00025 \\
\hline beta1[1] & 0.41111 & 0.40000 & 0.40007 & 0.33320 & 0.50013 \\
\hline beta1[2] & 1.00000 & 1.00000 & 1.00000 & 0.99990 & 1.00010 \\
\hline beta1[3] & 1.00000 & 1.00000 & 1.00000 & 0.99989 & 1.00011 \\
\hline Sigma & 0.00064 & 0.00063 & 0.00063 & 0.00063 & 0.00065 \\
\hline Nu & 0.20606 & 0.20563 & 0.20416 & 0.18079 & 0.23264 \\
\hline
\end{tabular}

\subsection{Interpreting the posterior distributions}

Now we will discuss the results of the analysis. The parameter estimates in Table 1 indicates that the most credible value of the slope $\beta_{1,1}$ is about 0.4 , which means that $\mathrm{KGW}$ in $1^{\text {st }}$ month after surgery increases about $0.4 \mathrm{~mm}$ for every $1-\mathrm{mm}$ increase in $\mathrm{KGW}$ at baseline. The posterior distribution of $\beta_{1,2}$ shows that the most credible value of the slope $\beta_{1,2}$ is about 1 , which means that $\mathrm{KGW}$ in $3^{\text {rd }}$ month after surgery increases about $1 \mathrm{~mm}$ for every $1-\mathrm{mm}$ increase in $\mathrm{KGW}$ in $1^{\text {st }}$ month. The slope $\beta_{1,3}$ has modal estimate of about 1 . It means that $\mathrm{KGW}$ in $6^{\text {th }}$ month after surgery increases about $1 \mathrm{~mm}$ for every $1-\mathrm{mm}$ increase in $\mathrm{KGW}$ in $3^{\text {rd }}$ month.One way to summarize the uncertainty is by marking the span of values that are most credible and cover 95\% of the distribution. This is the highest density interval (HDI). Values within the 95\% HDI are more credible (i.e., have higher probability "density") than values outside the HDI, and the values inside the HDI have a total probability of $95 \%$. Given the 118 data points, the $95 \%$ HDI for the slope $\beta_{1,1}$ goes from a slope of about 0.33 $\mathrm{mm}$ per $\mathrm{mm}$ to a slope of about $0.5 \mathrm{~mm}$ per mm. The $95 \%$ HDI for both slopes $\beta_{1,2}$ and $\beta_{1,3}$ go from a slope of about 0.99 to a slope of about 1.The last two HDIs are narrower. The estimates of the slopes $\beta_{1,2}$ and $\beta_{1,3}$ are very precise. The individuals seem to have nearly the same slope and $\sigma_{l}$ is estimated to be small (see Table 1). In fact, the $95 \%$ HDIs on the slopes are far from zero. Thus, there is no doubt that there is a linear trend in these KGW data.

\section{Conclusion}

We have studied the behavior of the clinical periodontal parameter keratinized gingiva width (KGW) during the gingival recession therapy. This study presents an approach to study the rate of increase of the keratinized gingiva width (KGW) during treatment. The slope parameters in the model are meaningful because they describe tendencies in the KGW data.

\section{References}

[1] Iv. Chenchev, D. Atanasov, D. Vicheva, The treatment of gingival recessions - Our experience, Romanian Journal of Rhinology, 2016, 6 (22): 85-91.

[2] J. Weinmann, Progress of gingival inflammation into the supporting structures of the teeth, J Periodontol, 1941, 12: 71-82

[3] C. Ochsenbein, Newer concept of mucogingival surgery, J Periodontol, 1960, 31: 175-185.

[4] U. Hangorsky, NF. Bissada, Clinical assessment of free gingival graft effectiveness on the maintenance of periodontal health, $J$ Periodonol, 1980, 51: 274-278.

[5] NP. Lang, H. Löe, The relationship between the width of keratinized gingiva and gingival health, J Periodontol, 1972, 43(10): 623627.

[6] FA. Carrenza, JJ. Carraro, Mucogingival techniques in periodontal surgery, J Periodontol, 1970, 41: $294-299$.

[7] J. Ainamo, A. Talari, The increase with age of the width of attached ginigva, J Periodontal Res, 1976,11:182.

[8] PM. Camargo, PR. Melnick, EG. Kenney, The use of free gingival grafts for aesthetic purposes, Periodontal 2000, 2001, 27 : 72- 6.

[9] M. Miyasato, M. Crigger, J. Egelberg, Gingival condition in areas of minimal and appreciable width of keratinized gingival, $J$ Clin Periodontol, 1977, 4(3):200-9.

[10] RJ. Harris, The connective tissue with partial thickness double pedicle graft: The results of 100 consecutively treated defects, $J$ Periodontol, 1994, 65:448-461.

[11] M. Nevis, Attached gingiva- mucogingival therapy and restorative dentistry, Int J Periodontics Restorative Dent, 1986 ; 6: 9-37.

[12] JJ. Massad, JF. Anderson, Hamular frenum modification: a removable denture prosthesis retention and stability enhancement, Int $J$ Periodontics Restorative Dent, 2001, 21: 183-189.

[13] PD. Jr Miller, Root coverage using the free soft tissue autograft following citric acid application: III. A successful and predictable procedure in areas of deep-wide recession, Int J Periodontics Restorative Dent, 1985, 5:14-37.

[14] A. Ainamo, Influence of age on the location of the maxillary Mucogingival Junction, J Periodont Res, 1978, 13:189.

[15] A. Ainamo, J. Ainamo, The width of attached gingiva on Supraerupted teeth, J Periodont Res, 1978; 13:194. 
[16] I. Ericsson, J. Lindhe, Recession in sites with inadequate width of the keratinized gingiva. An experimental study in the dog, Journal of Clinical Periodontology,1984, 11:95-103.

[17] L. Chambrone, D. Chambrone, FE. Pustiglioni, LA. Chambrone, LA. Lima, Can subepithelial connective tissue grafts be considered the gold standard procedure in the treatment of Miller Class I and II recession-type defects? Journal of Dentistry, 2008, 36: 659671 .

[18] V. Noncheva, M. Dobreva, Iv, Chenchev, Is Treatment of Gingival Recession Predictable? IOSR Journal of Mathematics (IOSRJM), e-ISSN: 2278-5728, p-ISSN: 231-765X. Volume12, Issue 5 Ver. V (Sep. -Oct.2016), pp. 07-11.

[19] Iv. Chenchev, D. Atanasov, D. Vicheva, V. Noncheva, Comparative Evaluation of the Subjective Results from the Treatment of Gingival Recessions with Connective Tissue Graft and Platelet Rich Fibrin Membrane, IOSR Journal of Dental and Medical Sciences, Vol. 15, Issue 5, Ver. 05 May, 2016, pp 73-78.

[20] A. Gelman, J.B. Carlin, H.S. Stern, D.B. Rubin, Bayesian data analysis (Third Edition, Boca Raton, Chapman and Hall-CRC, 2013).

[21] J.K. Kruschke, Doing Bayesian data analysis: A tutorial with $R$ and BUGS (Second Edition. Burlington, MA: Academic Press/Elsevier, 2011).

[22] WB. Hall, Prevention of recession, in "Decision Making in Periodontology, (WB. Hall ed, Mosby, St. Louis, 1998), pp 110-111.

[23] Wennstrom J, Prato GP, Mucogingiva therapy, In "Clinical Periodontology and Implant Dentistry", (3rd ed, Lindhe J ed, Munksgaard, Copenhagen. 1997), pp. 550-596.

[24] M. Dobreva, V. Noncheva, Iv. Chenchev, Bayesian approach for the comparison of two methods of treatment, Proceedings of International Scientific Jubilee Conference 25 years Faculty of Mathematics and Informatics, University of Veliko Turnovo "St. Cyril and Methodius", Bulgaria, November, 2015, pp 107-112.

[25] M. Plummer, JAGS: A Program for Analysis of Bayesian Graphical Models Using Gibbs Sampling, Proceedings of the 3rd International Workshop on Distributed Statistical Computing (DSC 2003), March 20-22, 2003, Vienna, Austria, ISSN 1609-395X. 